\title{
Evaluation of disease severity in mucopolysaccharidoses
}

\author{
Michael Beck $^{\mathrm{a}, *}$, Joseph Muenzer ${ }^{\mathrm{b}}$ and Maurizio Scarpa ${ }^{\mathrm{c}}$ \\ ${ }^{a}$ Children's Hospital, University of Mainz, Mainz, Germany \\ ${ }^{\mathrm{b}}$ Department of Pediatrics, University of North Carolina, Chapel Hill, NC, USA \\ ${ }^{\mathrm{c}}$ Department of Pediatrics, University of Padova, Italy
}

\begin{abstract}
The mucopolysaccharidoses (MPS) are a group of lysosomal storage disorders characterized by a wide variation in symptoms and progression rates. Each of the MPS types is caused by a deficiency in one of the enzymes involved in the catabolic pathway of glycosaminoglycans. MPS are usually suspected on the basis of the concomitant presence of several typical features of the disease, such as short stature, coarse facial features, loss of hearing, bone deformities, joint stiffness, organomegaly, respiratory and cardiovascular complications, and, in some cases, developmental delay. Very reduced or absent enzyme activity is required to confirm an MPS diagnosis. In the past, treatment of MPS was limited to the management of individual symptoms of the diseases. Currently, specific therapies such as hematopoietic stem cell transplantation and enzyme replacement therapy have improved the outlook for many MPS patients. To monitor disease progression and the impact of therapy in these patients, there is need for a validated scoring system for evaluating disease severity in MPS. A scoring system should take into account all aspects of MPS, particularly quality of life and functioning of arms, hands and legs and related mobility, endurance, self care and social functioning. This paper discusses several of the available scoring systems for bone/skeletal disorders and their potential usefulness in patients with MPS.
\end{abstract}

Keywords: Diagnosis, glycosaminoglycan, mucopolysaccharidoses, severity of illness index, treatment

\section{Introduction}

The mucopolysaccharidoses (MPS) are a group of heritable lysosomal storage disorders caused by a deficiency in glycosaminoglycan (GAG)-degrading enzymes (Table 1). The resulting accumulation of undegraded or partly degraded GAGs in lysosomes causes progressive tissue and organ dysfunction. All MPS types are very rare disorders with prevalence data varying considerably between regions. Birth estimates for MPS VI, for example, vary from about 1 in 40,000 births in Turkish immigrants living in Germany to about 1 in 1,500,000 births in Sweden, making up $4 \%$ of all

*Address for correspondence: Michael Beck, Children's Hospital, University of Mainz, Department of Pediatrics, Langenbeckstr. 2, 55131 Mainz, Germany. Tel.: +49 613117 2398; Fax: +496131 17 5672; E-mail: beck@kinder.klinik.uni-mainz.
MPS diseases in both studies [3,21]. As birth prevalence estimates are based on clinical identification, true birth prevalence of the MPS will not be obtained until newborn screening for MPS becomes available.

The MPS have a chronic and progressive course. Each MPS type can be caused by a variety of mutations in the same enzyme, which is reflected in a wide spectrum of progression rates and clinical phenotypes within each enzyme deficiency (Fig. 1). Life expectancies vary between and within the different MPS types, but are generally markedly reduced. Typical features of MPS (with the exception of MPS III) include short stature, coarse facial features, loss of hearing, organomegaly, and respiratory and cardiovascular complications. MPS III and VII and severe forms of MPS I (Hurler and Hurler/Scheie disease) and MPS II are characterized by mental retardation (Table 1). Most MPS types with the exception of MPS III are also asso- 
Table 1

Classification of mucopolysaccharidoses

\begin{tabular}{|c|c|c|c|c|c|}
\hline Number & Eponym & Mental status & Skeletal manifestations & Enzyme deficiency & GAG affected \\
\hline MPS IH & Hurler & Mental retardation & $\begin{array}{l}\text { Short stature, } \\
\text { dysostosis multiplex }\end{array}$ & $\alpha$-L-Iduronidase & $\begin{array}{l}\text { Dermatan sulfate, } \\
\text { heparan sulfate }\end{array}$ \\
\hline MPS IS & Scheie & Normal intelligence & Dysostosis multiplex & $\alpha$-L-Iduronidase & $\begin{array}{l}\text { Dermatan sulfate, } \\
\text { heparan sulfate }\end{array}$ \\
\hline MPS IH/IS & Hurler-Scheie & $\begin{array}{l}\text { Phenotype intermediate } \\
\text { between IH and IS }\end{array}$ & $\begin{array}{l}\text { Phenotype intermediate } \\
\text { between IH and IS }\end{array}$ & $\alpha$-L-Iduronidase & $\begin{array}{l}\text { Dermatan sulfate, } \\
\text { heparan sulfate }\end{array}$ \\
\hline MPS II (severe) & Hunter (severe) & Mental retardation & $\begin{array}{l}\text { Short stature, } \\
\text { dysostosis multiplex }\end{array}$ & Iduronate sulfatase & $\begin{array}{l}\text { Dermatan sulfate, } \\
\text { heparan sulfate }\end{array}$ \\
\hline MPS II (mild) & Hunter (mild) & Normal intelligence & $\begin{array}{l}\text { Short stature, } \\
\text { dysostosis multiplex }\end{array}$ & Iduronate sulfatase & $\begin{array}{l}\text { Dermatan sulfate, } \\
\text { heparan sulfate }\end{array}$ \\
\hline MPS IIIA & Sanfilippo A & $\begin{array}{l}\text { Profound mental } \\
\text { retardation }\end{array}$ & $\begin{array}{l}\text { Relatively mild } \\
\text { somatic manifestations }\end{array}$ & $\begin{array}{l}\text { Heparan } N \text {-sulfatase } \\
\text { (sulfamidase) }\end{array}$ & Heparan sulfate \\
\hline MPS IIIB & Sanfilippo B & Similar to IIIA & Similar to MPS IIIA & $\begin{array}{l}\alpha-N \text {-Acetyl- } \\
\text { glucosaminidase }\end{array}$ & Heparan sulfate \\
\hline MPS IIIC & Sanfilippo C & Similar to IIIA & Similar to MPS IIIA & $\begin{array}{l}\text { Acetyl-CoA: } \alpha- \\
\text { glucosaminide } \\
\text { acetyltransferase }\end{array}$ & Heparan sulfate \\
\hline MPS IIID & Sanfilippo D & Similar to IIIA & Similar to MPS IIIA & $\begin{array}{l}\text { N-Acetylglucosamine } \\
\text { 6-sulfatase }\end{array}$ & Heparan sulfate \\
\hline MPS IVA & Morquio A & Normal intelligence & $\begin{array}{l}\text { Short-trunk dwarfism, } \\
\text { skeletal manifestations } \\
\text { distinct from } \\
\text { other MPS types }\end{array}$ & $\begin{array}{l}\text { Galactose 6-sulfatase } \\
\text { ( } N \text {-acetylgalactosamine } \\
\text { 6-sulfatase) }\end{array}$ & $\begin{array}{l}\text { Keratan sulfate, } \\
\text { chondroitin } 6 \text {-sulfate }\end{array}$ \\
\hline MPS IVB & Morquio B & Similar to MPS IVA & Similar to MPS IVA & $\beta$-Galactosidase & Keratan sulfate \\
\hline MPS VI & Maroteaux-Lamy & Normal intelligence & $\begin{array}{l}\text { Short stature, } \\
\text { dysostosis multiplex }\end{array}$ & $\begin{array}{l}N \text {-Acetylgalactosamine } \\
\text { 4-sulfatase } \\
\text { (arylsulfatase B) }\end{array}$ & Dermatan sulfate \\
\hline MPS VII & Sly & Mental retardation & $\begin{array}{l}\text { Short stature, } \\
\text { dysostosis multiplex }\end{array}$ & $\beta$-glucuronidase & $\begin{array}{l}\text { Dermatan sulfate, } \\
\text { heparan sulfate } \\
\text { chondroitin 4-, } \\
\text { 6-sulfates }\end{array}$ \\
\hline MPS IX & - & Normal intelligence & Short stature & Hyaluronidase & Hyaluronan \\
\hline
\end{tabular}

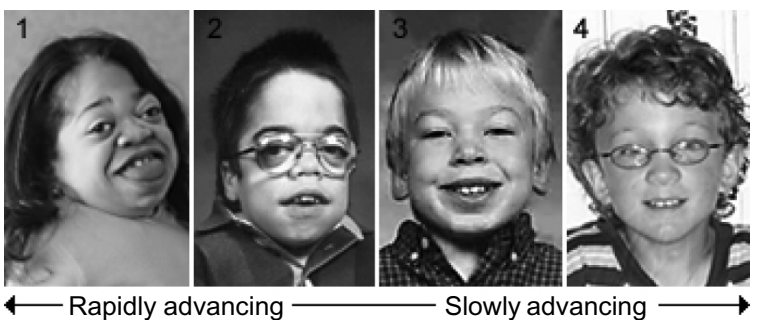

Fig. 1. Spectrum of disease severity in MPS VI. Photographs showing four children with MPS VI aged 9-12 years. Reprinted with permission from The National MPS Society, Inc.

ciated with widespread skeletal abnormalities and joint disease.

Very apparent bone deformities that often occur in MPS patients include severe abnormalities of the spine such as kyphosis (gibbus deformity) and scoliosis, hip dysplasia, genu valgum (knock knees), and claw hands. The MPS have in common a characteristic radiologic expression referred to as dysostosis multiplex, which manifests as changes in the skull, thorax, pelvis, hands and spine [24]. Dysostosis multiplex has been de- scribed in patients with MPS I, MPS II, MPS III, MPS IV, MPS VI, MPS VII, but also in patients with mucolipidoses and other storage diseases such as multiple sulphatase deficiency and GM1 gangliosidosis. MPS III patients usually do not show severe somatic manifestations (Table 1).

\section{Diagnosis and treatment of MPS}

MPS are usually suspected on the basis of several typical signs and symptoms occurring in concert. Severe cases are generally recognized early in life, but for patients with more attenuated phenotypes, it can take many years before their symptoms are recognized as being of metabolic origin. The first method that was used to distinguish between different MPS types was analysis of urinary GAG. However, this technique cannot discriminate between different MPS subtypes (e.g. the different subtypes of MPS III) and may fall in the normal range for the attenuated or slowly progressing forms. A definitive diagnosis can only be made by 
measuring enzyme activity, which is generally done in cultured fibroblasts, leukocytes, or serum depending on the enzyme measured, specific assay and laboratory preference. A very reduced or absent enzyme activity together with clinical signs of the disease establishes the presence of the disease [14,22,33]. The diagnosis can be supported by mutational analysis of the affected gene, which also provides valuable information for genetic counseling of the patients and their families. In MPS II, which is an X-linked disease, it is the only way to identify female carriers with certainty [22]. The introduction of new, specific therapies for several MPS types has increased the need for early diagnosis.

Initially the treatment of MPS was limited to palliative care, involving physical therapy and the treatment or alleviation of individual symptoms. A better understanding of the pathophysiology and the natural history of these disorders has led to the development of diseasespecific therapies that aim at slowing down progression. These therapies are based on the assumption that the replacement or delivery of the missing enzyme will result in normal GAG metabolism and reduce and/or prevent further lysosomal storage and dysfunction. An enzyme can be replaced either indirectly through transplanted donor cells (hematopoietic stem cell transplantation [HSCT]), directly (enzyme replacement therapy [ERT]), or through autologous cells genetically modified to express the enzyme (gene therapy). HSCT has been applied in several MPS types with variable success $[16,20]$ and is the procedure of choice to prevent brain disease in severe MPS IH when performed early in life. This positive effect is believed to be related to migration of donor-derived cells into the CNS. The donor-derived macrophages and microglia can become a source of local enzyme which could cross-correct defective cells in the CNS. Similar success has not been documented for the other MPS with primary CNS disease (MPS II or III). Moreover, the use of this therapy is limited by the mortality risk and the need for suitable stem cell donors.

ERT uses human enzyme manufactured by recombinant DNA technology in high-output cell lines. It is now approved for clinical practice and recommended treatment for MPS I, II, and VI [10,14,31,33]. The recombinant enzymes are approved for intravenous administration, but intrathecal and intraarticular administration of enzyme are currently being investigated as a means to circumvent the low accessibility of enzyme to the central nervous system and articular cartilage when administered intravenously. Both techniques have recently shown promising results in MPS VI cats $[1,2]$ and MPS I dogs [8] and have sporadically been applied in humans. Munoz-Rojas et al [23] described the case of a patient with MPS I who was treated with intrathecal ERT [23]. Promising results have also been reported in studies using ERT to treat patients before and possibly through the HCST engraftment period although no controlled or blinded trials have been reported [34].

Gene therapy for MPS is still in an experimental stage [4]. Important hurdles that need to be overcome before gene therapy is ready to be used in clinical practice include adequate and sustained expression of the gene in the right cell type and tissue. Another concern remains the safety of this technique [25].

\section{Evaluation of disease severity}

Together with the improving outlook for many MPS patients due to the introduction of disease-specific therapies, there is a need for a disease severity scoring system to evaluate disease progression, prognosis, and most importantly, response to therapy. Ideally, a scoring system for the evaluation of disease severity should also quantify the burden of the disease at a certain time point and should be able to predict the future evolution of the disease [5]. Such diagnostic instrument, for example, may help to decide whether a child with MPS I has the severe form (Hurler disease) and should undergo HSCT, or is affected by the attenuated form (Scheie disease) and should better receive ERT. An appropriate scoring system could also be applied to study the progression of the disease and to quantify the rate of deterioration for some clinical parameters. Such diagnostic instrument has been developed for example for patients affected by Niemann-Pick C disease [35].

Other requirements for a good scoring system are disease-specificity, reliability of repeated test results, and validity across countries, especially when it concerns very rare diseases such as the MPS. Finally, the scoring system should be easy to use and contain only a few parameters that can be easily and readily obtained so that it can be used in small centers. The development of a scoring system for MPS can be guided by the experience with existing scoring systems developed for other disorders. In the following paragraphs, we discuss different types of scoring systems that could be used in assessing disease severity in MPS.

\subsection{Biomarkers}

Genotype-phenotype correlation studies have shown that allelic heterogeneity reflects the clinical hetero- 
geneity in the MPS, suggesting that the amount of residual enzyme activity affects the clinical course of these diseases [12,17,30]. However, in most cases, gene analysis, expression studies and transcript analysis are not sufficient to predict a patient's phenotype.

The only objective biomarker currently available for assessing disease severity in MPS is urinary GAG. In MPS VI, an international cross-sectional survey in 121 patients suggested that urinary GAG levels can predict clinical morbidity [29]. High urinary GAG levels $(>200 \mu \mathrm{g} / \mathrm{mg}$ creatinine) were shown to reflect rapidly progressive disease, which is characterized by short stature (Fig. 2a), low body weight, impaired endurance (Fig. 2b), compromised pulmonary function, and reduced joint range of motion (Fig. 2c). Patients with GAG levels $<100 \mu \mathrm{g} / \mathrm{mg}$ creatinine were shown to have better survival than those with GAG levels $\geqslant$ $100 \mu \mathrm{g} / \mathrm{mg}$ creatinine.

Some other targets have been identified in blood and urine, but their usefulness as biomarkers of disease activity for MPS needs to be confirmed. Studies have shown that serum levels of heparin cofactor II-thrombin (HCII-T) complex reflect disease severity and responsiveness to therapy [26,27]. A promising technique for monitoring patients with MPS involves the derivatization of urinary oligosaccharides and subsequent analysis by electrospray ionization-tandem mass spectrometry [13]. Although more research is warranted, this technique could be a sensitive method for evaluating the impact of therapy regimens and possibly for making phenotype predictions $[6,13]$. Specific oligosaccharide profiles have been identified for all MPS types except for MPS IIIB and IIIC [13].

\subsection{Quantitative measurements}

Quantitative measurements such as height and head circumference can reflect disease severity in several MPS types. This was clearly shown in a recently published report of the Hunter Outcome Survey [32]. This international, multicenter, long-term observational survey collects different data from patients with MPS II in an attempt to obtain better insight into the natural history / progression of this disorder and to assess the long-term impact of ERT in children with the disease. As of May 2007, 263 patients had already been enrolled in the survey. Growth data show that patients with MPS II are often very tall for their age in their first 2 or 3 years of life. At the age of 7 to 10 years, growth rate slows down, ultimately resulting in heights below the third percentile [32]. Severely affected patients seem to have a bigger head circumference at a young age than those who are less severely affected. The latter was previously observed in a clinical study in 77 MPS II patients [28].

\subsection{Quantitative imaging}

Bone disease in MPS may also be quantified using magnetic resonance imaging (MRI). A semiquantitative scoring system, the MRI Bone Marrow Burden (BMB) score, was previously developed for Gaucher disease for measuring bone marrow abnormalities and bone marrow invasion in a semi-quantitative way [19]. The BMB evaluates bone marrow displacement, whereas in MPS disease, the major problem is bone deformity. It remains unclear whether a similar scoring system could also be developed for MPS. Disadvantages of using MRI for measuring disease severity are that it does not give any information about function and that it is expensive and not easy to implement in clinical practice.

\subsection{Measurement of degree of deformities}

Another possibility for evaluating clinical severity in MPS is a scoring system based on the presence of bone deformities such as deformity of the spinal column, claw hands, hip dysplasia, etc., with each deformity counting for a certain number of points. Higher scores reflect more severe deformities. This approach is used in a part of a scoring system that is currently being developed by Schulze Frenking from Mainz in Germany for MPS II (unpublished). However, this scoring system needs further validation before it can be used in clinical practice. A disadvantage of this system is that it only quantifies the severity of bone deformities, but does not give any information about the impact of these deformities on the patient's functioning.

\subsection{Functional tests}

Probably the most valuable way to measure clinical disease severity is the evaluation of the patient's functioning. Some functional tests have been developed specifically for skeletal diseases. A very well known and validated test in this respect is the International Classification of Functioning, Disability and Health (ICF) (http://www.who.int/classifications/icfbrowser). This test consists of several health and health-related domains that are classified in two lists, one covering body functions and structure, and one covering activi- 

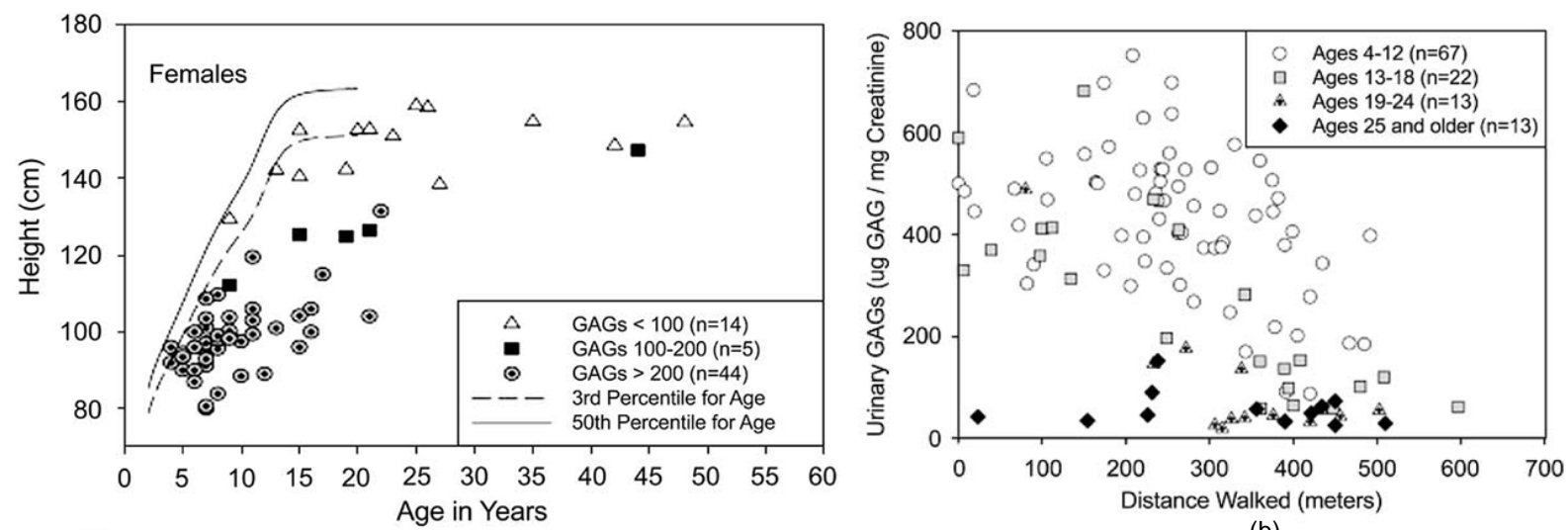

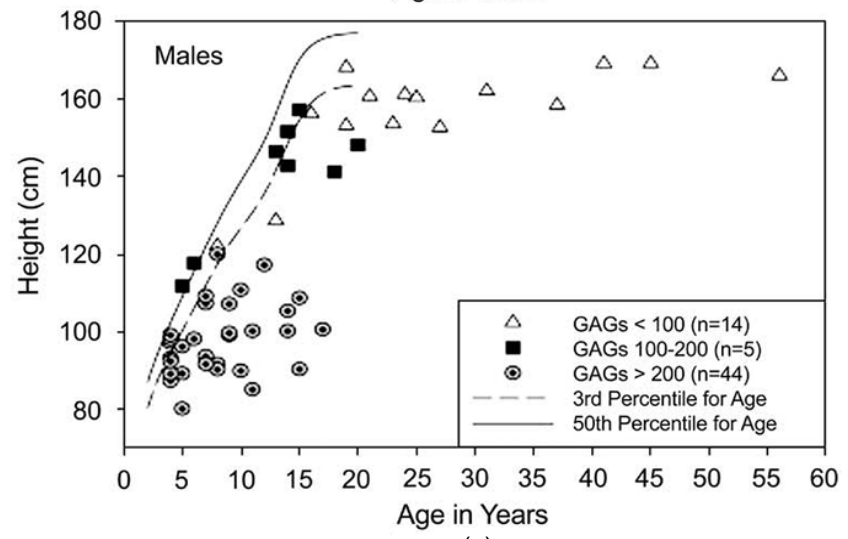

(a)

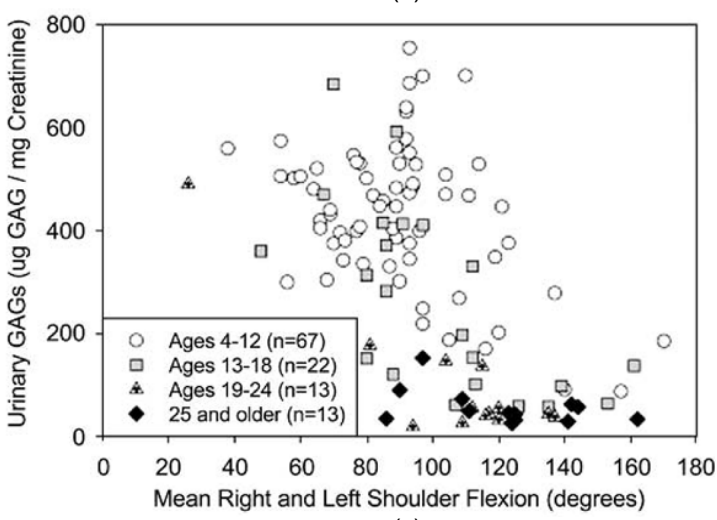

(c)

Fig. 2. High urinary GAG levels ( $>200 \mu \mathrm{g} / \mathrm{mg}$ creatinine) reflect (a) short stature, (b) impaired endurance (distance walked in a 6-min walk test), and (c) reduced joint range of motion (right and left shoulder flexion) in MPS VI patients. Reprinted from Swiedler et al. [15], with permission from John Wiley \& Sons, Inc.

ty and participation. Since an individual's functioning and disability occurs in a context, the ICF also includes a list of environmental factors. A disadvantage of this test is that it involves many complicated tasks that take a long time to complete; therefore, it is not considered practical for daily assessment of function.

A more practical functional test is the Pediatric Evaluation of Disability Inventory (PEDI). The PEDI is a standardized questionnaire for the evaluation of capability and performance in children from 6 months to 7.5 years in three categories: self care, mobility, and social function. It was developed in 1992 by Haley et al from Boston University, USA, and is available as a paper and electronic version, with normative data for half-year age groups (http://www. bu.edu/hdr/products/pedi/index.html). The PEDI can be administered by professional judgment of clinicians and educators who are familiar with the child, or by structured interview of the parent. The amount of time required for the parent interview is about 45 minutes. The PEDI has been successfully applied in patients with osteogenesis imperfecta. In a study by Engelbert et al [11], the PEDI revealed a statistically significant difference in mobility between patients with the mild form of the disease and those with the severe form [11]. A more recently published study in patients with osteogenesis imperfecta showed that the PEDI can also be used to assess the impact of treatment on functional health [18]. One year of treatment with intravenous pamidronate therapy, resulted in a significant improvement in the self care and mobility categories in these patients. The PEDI is believed to be applicable for several skeletal disorders and may therefore also be a valuable tool to assess patients with MPS.

Another functional test that is well-known is the POSNA (Pediatric Orthopedic Society of North America) pediatric musculoskeletal functional health questionnaire. This questionnaire assesses upper extremity function, transfers and mobility, physical function and sports, comfort (pain-free time), happiness and satisfaction, and expectations for treatment [7]. All scores range from 0 to 100 , with 100 representing best func- 
tioning, least pain, etc. The POSNA global scale is calculated by averaging the upper extremity function, transfers and mobility, physical function and sports, and comfort scores. The POSNA scale is a reliable and validated scoring system that is easy to use and has been applied in patients with 138 different primary orthopedic diagnoses and a wide variety of ages. It encompasses all severity levels of many skeletal disorders. Another advantage of this questionnaire is that it can be completed either by the child or by one of his or her parents. It takes 30-45 minutes to complete.

A few years ago, the MPS physical performance measure (MPS-PPM), a special functional test, was developed for MPS I patients [9]. The MPS-PPM is an easy-to-use test that includes arm function (seven tasks), leg function (four tasks), and endurance (two tasks) (Table 2). For evaluating arm and leg function, subjects were given scores for performance $(0=$ physically unable to complete the task or unable to complete the task in $<1 \mathrm{~min} ; 1=$ able to complete the task in $<$ $1 \mathrm{~min}$ ) and speed of task completion. Speed was not recorded for hand raising. Range of shoulder flexion was measured using an electronic goniometer (performance score $0=$ unable to raise shoulder $>90^{\circ}, 1=$ able to raise shoulder $>90^{\circ}$ ). Endurance was tested by measuring the distance covered and the subject's maximum heart rate during a 3-min walk at comfortable or fast speed. The total severity score was the sum of all item scores. Correlation analyses revealed a statistically significant correlation of the MPS-PPM score with age $(p=0.019)$. Leg function appeared to be best correlated with the total severity score $(p=0.007)$. The MPS-PPM can possibly be used to evaluate the severity of MPS I and to assess the impact of therapy. However, further validation of the MPS-PPM for application to other MPS and the development of normative scores for rating performance and speed are warranted.

\section{Conclusions}

With the advent of new therapies such as HSCT and ERT for several MPS types, there is increasing need for standardized methods for monitoring the progression of patients with MPS and their response to therapy. The development of an effective scoring system is essential in this respect. More research is warranted to elucidate whether disease severity in MPS patients can be adequately measured using currently available scoring systems such as PEDI and POSNA, or whether this requires MPS-specific severity scores. A number of scor-

\section{Table 2}

The MPS physical performance measure (MPS-PPM), developed for patients with MPS I [9]

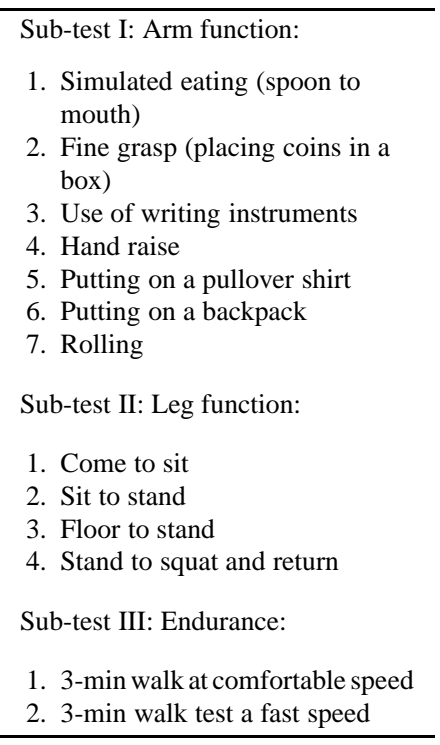

ing systems for MPS are under development, but these need further validation before they can be applied in clinical practice. The validation of a scoring system for rare diseases such as the MPS can be a challenge due to the low number of affected patients who are generally geographically dispersed. Ideally, a scoring system for MPS should encompass all aspects of the diseases, particularly functioning (physical functioning and related mobility/endurance, self care and social functioning) and quality of life, which are considered most essential for MPS patients. However, it remains unclear whether it is feasible to develop a scoring system that applies for all MPS types and/or combines functioning and QoL in one single test.

\section{Acknowledgements}

This manuscript was developed as the result of a meeting of experts entitled "Promoting Bone Health in MPS VI: Framing New Therapies" held in Oakland, California in October, 2008. Editorial assistance in the development of this manuscript was provided by Ismar Healthcare NV, Belgium, and was paid for by BioMarin Pharmaceutical Inc., Novato, CA. BioMarin reviewed the manuscript to insure the accuracy of all statements regarding enzyme replacement therapy with galsulfase, but had no role in the content presented and discussed at the meeting. All authors participated in the development and writing of the manuscript and are fully responsible for its content. 


\section{Conflicts of interest}

Michael Beck and Maurizio Scarpa have received unrestricted research grants, honoraria and travel support from Genzyme, Shire, BioMarin and Actelion.

\section{References}

[1] D. Auclair, L.K. Hein, J.J. Hopwood and S. Byers, Intraarticular enzyme administration for joint disease in feline mucopolysaccharidosis VI: enzyme dose and interval, Pediatr Res 59 (2006), 538-543.

[2] D. Auclair, J.J. Hopwood, J.F. Lemontt, L. Chen and S. Byers, Long-term intra-articular administration of recombinant human $N$-acetylgalactosamine-4-sulfatase in feline mucopolysaccharidosis VI, Mol Genet Metab 91 (2007), 352361.

[3] F. Baehner, C. Schmiedeskamp, F. Krummenauer, E. Miebach, M. Bajbouj, C. Whybra, A. Kohlschutter, C. Kampmann and M. Beck, Cumulative incidence rates of the mucopolysaccharidoses in Germany, J Inherit Metab Dis 28 (2005), 10111017.

[4] M. Beck, New therapeutic options for lysosomal storage disorders: enzyme replacement, small molecules and gene therapy, Hum Genet 121 (2007), 1-22.

[5] M. Beck and U. Widmer, Assessing multiorgan disease severity: scoring systems and applicability in LSDs, Acta Paediatr Suppl 95 (2006), 41-42.

[6] A. Crawley, S.L. Ramsay, S. Byers, J. Hopwood and P.J. Meikle, Monitoring dose response of enzyme replacement therapy in feline mucopolysaccharidosis type VI by tandem mass spectrometry, Pediatr Res 55 (2004), 585-591.

[7] L.H. Daltroy, M.H. Liang, A.H. Fossel and M.J. Goldberg, The POSNA pediatric musculoskeletal functional health questionnaire: report on reliability, validity, and sensitivity to change. Pediatric Outcomes Instrument Development Group. Pediatric Orthopaedic Society of North America, J Pediatr Orthop 18 (1998), 561-571.

[8] P. Dickson, M. McEntee, C. Vogler, S. Le, B. Levy, M. Peinovich, S. Hanson, M. Passage and E. Kakkis, Intrathecal enzyme replacement therapy: successful treatment of brain disease via the cerebrospinal fluid, Mol Genet Metab 91 (2007), 61-68.

[9] H.M. Dumas, M.A. Fragala, S.M. Haley, A.M. Skrinar, J.E. Wraith and G.F. Cox, Physical performance testing in mucopolysaccharidosis I: a pilot study, Pediatr Rehabil 7 (2004), 125-131.

[10] R.P. El Dib and G.M. Pastores, Laronidase for treating mucopolysaccharidosis type I, Genet Mol Res 6 (2007), 667-674.

[11] R.H.H. Engelbert, J.W.H. Custers, J. van der Net, Y. van der Graaf, F.A. Beemer and P.J.M. Helders, Functional outcome in osteogenesis imperfecta: disability profiles using the PEDI, Pediatr Phys Ther 9 (1997), 18-22.

[12] R. Froissart, I. Moreira da Silva, N. Guffon, D. Bozon and I. Maire, Mucopolysaccharidosis type II-genotype/phenotype aspects, Acta Paediatr Suppl 91 (2002), 82-87.

[13] M. Fuller, T. Rozaklis, S.L. Ramsay, J.J. Hopwood and P.J. Meikle, Disease-specific markers for the mucopolysaccharidoses, Pediatr Res 56 (2004), 733-738.

[14] R. Giugliani, P. Harmatz and J.E. Wraith, Management guidelines for mucopolysaccharidosis VI, Pediatrics 120 (2007), $405-418$.
[15] F.S. Haddad, D.H.A. Jones, A. Vellodi, N. Kane and M.C. Pitt, Carpal tunnel syndrome in the mucopolysaccharidoses and mucolipidoses, J Bone Joint Surg Br 79 (1997), 576-582.

[16] W. Krivit, Allogeneic stem cell transplantation for the treatment of lysosomal and peroxisomal metabolic diseases, Springer Semin Immunopathol 26 (2004), 119-132.

[17] T. Litjens, D.A. Brooks, C. Peters, G.J. Gibson and J.J. Hopwood, Identification, expression, and biochemical characterization of $N$-acetylgalactosamine-4-sulfatase mutations and relationship with clinical phenotype in MPS-VI patients, $A m$ J Hum Genet 58 (1996), 1127-1134.

[18] K. Löwing, E. Åström, K.A. Oscarsson, S. Söderhäll and A.C. Eliasson, Effect of intravenous pamidronate therapy on everyday activities in children with osteogenesis imperfecta, Acta Paediatr 96 (2007), 1180-1183.

[19] M. Maas, C. van Kuijk, J. Stoker, C.E.M. Hollak, E.M. Akkerman, J.F.M.G. Aerts and G.J. den Heeten, Quantification of bone involvement in Gaucher disease: MR imaging bone marrow burden score as an alternative to Dixon quantitative chemical shift MR imaging-initial experience, Radiology 229 (2003), 554-561.

[20] J.J. Malatack, D.M. Consolini and E. Bayever, The status of hematopoietic stem cell transplantation in lysosomal storage disease, Pediatr Neurol 29 (2003), 391-403.

[21] G. Malm, A.M. Lund, J.E. Månsson and A. Heiberg, Mucopolysaccharidoses in the Scandinavian countries: incidence and prevalence, Acta Paediatr 97 (2008), 1577-1581.

[22] R. Martin, M. Beck, C. Eng, R. Giugliani, P. Harmatz, V. Muñoz and J. Muenzer, Recognition and diagnosis of mucopolysaccharidosis II (Hunter syndrome), Pediatrics 121 (2008), e377-e386.

[23] M.V. Munoz-Rojas, T. Vieira, R. Costa, S. Canani, A. John, L.B. Jardim, L.M. Vedolin, M. Raymundo, P.I. Dickson, E. Kakkis and R. Giugliani, Intrathecal enzyme replacement therapy in a patient with mucopolysaccharidosis type I and symptomatic spinal cord compression, Am J Med Genet A 146A (2008), 2538-2544.

[24] E.F. Neufeld and J. Muenzer, The mucopolysaccharidoses, in: The Metabolic and Molecular Bases of Inherited Disease, C.R. Scriver, A.L. Beaudet, W.S. Sly and D. Valle, eds, McGrawHill Medical Publishing Division, New York, 2001, pp. 34213452.

[25] K.P. Ponder and M.E. Haskins, Gene therapy for mucopolysaccharidosis, Expert Opin Biol Ther 7 (2007), 1333-1345.

[26] D.R. Randall, K.E. Colobong, H. Hemmelgarn, G.B. Sinclair, E. Hetty, A. Thomas, O.A. Bodamer, B. Volkmar, P.M. Fernhoff, R. Casey, A.K. Chan, G. Mitchell, S. Stockler, S. Melancon and T. Rupar, Heparin cofactor II-thrombin complex: a biomarker of MPS disease, Mol Genet Metab 94 (2008), 456461.

[27] D.R. Randall, G.B. Sinclair, K.E. Colobong, E. Hetty and L.A. Clarke, Heparin cofactor II-thrombin complex in MPS I: a biomarker of MPS disease, Mol Genet Metab 88 (2006), $235-243$.

[28] I.V.D. Schwartz, M.G. Ribeiro, J.G. Mota, M.B.P. Toralles, P. Correia, D. Horovitz, E.S. Santos, I.L. Monlleo, A.C. FettConte, R.P.O. Sobrinho, D.Y.J. Norato, A.C. Paula, C.A. Kim, A.R. Duarte and R. Boy, A clinical study of 77 patients with mucopolysaccharidosis type II, Acta Paediatr Suppl 96 (2007), 63-70.

[29] S.J. Swiedler, M. Beck, M. Bajbouj, R. Giugliani, I. Schwartz, P. Harmatz, J.E. Wraith, J. Roberts, D. Ketteridge, J.J. Hopwood, N. Guffon, M.C. Sa Miranda, E.L. Teles, K.I. Berger and C. Piscia-Nichols, Threshold effect of urinary gly- 
cosaminoglycans and the walk test as indicators of disease progression in a survey of subjects with Mucopolysaccharidosis VI (Maroteaux-Lamy syndrome), Am J Med Genet A 134 (2005), 144-150.

[30] N.J. Terlato and G.F. Cox, Can mucopolysaccharidosis type I disease severity be predicted based on a patient's genotype? A comprehensive review of the literature, Genet Med 5 (2003), 286-294.

[31] J.E. Wraith, The first 5 years of clinical experience with laronidase enzyme replacement therapy for mucopolysaccharidosis I, Expert Opin Pharmacother 6 (2005), 489-506.

[32] J.E. Wraith, M. Beck, R. Giugliani, J. Clarke, R. Martin and J. Muenzer, Initial report from the Hunter Outcome Survey, Genet Med 10 (2008), 508-516.

[33] J.E. Wraith, M. Scarpa, M. Beck, O.A. Bodamer, M.L. De, N Guffon, L.A. Meldgaard, G. Malm, A.T. Van der Ploeg and
J. Zeman, Mucopolysaccharidosis type II (Hunter syndrome): a clinical review and recommendations for treatment in the era of enzyme replacement therapy, Eur J Pediatr 167 (2008), 267-277.

[34] R.F. Wynn, J. Mercer, J. Page, T.F. Carr, S. Jones and J.E. Wraith, Use of enzyme replacement therapy (Laronidase) before hematopoietic stem cell transplantation for mucopolysaccharidosis I: experience in 18 patients, J Pediatr 154 (2009), 135-139.

[35] N.M. Yanjanin, J.I. Vélez, A. Gropman, K. King, S.E. Bianconi, S.K. Conley, C.C. Brewer, B. Solomon, W.J. Pavan, M. Arcos-Burgos, M.C. Patterson and F.D. Porter, Linear clinical progression, independent of age of onset, in Niemann-Pick disease, type C, Am J Med Genet B Neuropsychiatr Genet (2009) (doi: 10.1002/ajmg.b.30969). 